\title{
On-line extensible bin packing with unequal bin sizes
}

\author{
Deshi Yel and Guochuan Zhang $\beta^{\beta}$ \\ College of Computer Science, Zhejiang University, Hangzhou 310027, China.
}

received January 15, 2007, revised June 29, 2008, accepted February 9, 2008.

\begin{abstract}
In the extensible bin packing problem we are asked to pack a set of items into a given number of bins, each with an original size. However, the original bin sizes can be extended if necessary. The goal is to minimize the total size of the bins. We consider the problem with unequal (original) bin sizes and give the complete analysis on a list scheduling algorithm (LS). Namely we present tight bounds of LS for every collection of original bin sizes and every number of bins. We further show better on-line algorithms for the two-bin case and the three-bin case. Interestingly, it is proved that the on-line algorithms have better competitive ratios for unequal bins than for equal bins. Some variants of the problem are also discussed.
\end{abstract}

Keywords: On-line algorithms, extensible bin packing, competitive ratio

\section{Introduction}

We consider the following on-line extensible bin packing problem: there are $m$ bins $B_{1}, B_{2}, \ldots, B_{m}$ with original bin sizes $b_{1}, b_{2}, \ldots, b_{m}$. The original bin sizes can be extended if needed. The (final) size of a bin is defined to be the maximum of the original bin size and the total size of the items assigned to it. Items $\left\{a_{1}, a_{2}, \ldots, a_{n}\right\}$ arrive over list, which are not known in advance. When an item $a_{i}$ arrives it must immediately and irrevocably be assigned to one of the bins and the next item $a_{i+1}$ becomes known only after $a_{i}$ has been assigned. The size of item $a_{i}$ is $p_{i}$. The goal is to assign all items to the bins such that the total size of the bins is minimized.

This problem arises in a wide variety of contexts. It has many applications in bin packing, storage allocation and scheduling problems. Consider, for instance, a set of workers is given with regular working times. In case of needed, the worker can do some overtime works. The problem is to assign duties to the workers in such a way that the total work (regular working times plus overtime works) is minimized.

\footnotetext{
${ }^{\dagger}$ A preliminary version of this paper appeared in Proceedings of the First Workshop on Approximation and Online Algorithms (WAOA'03 235-247).

${ }^{\ddagger}$ Research was supported by NSFC(10601048) and National 973 Fundamental Research Project of China(No. 2007CB310900). E-mail: yedeshi@zju.edu.cn.

$\S$ Research was supported by NSFC (60573020). E-mail: zgc@zju.edu.cn.

1365-8050 @ 2009 Discrete Mathematics and Theoretical Computer Science (DMTCS), Nancy, France
} 
Competitive ratios. An instance of the problem consists of a list of items and $m$ bins with original sizes $b_{1}, b_{2}, \ldots, b_{m}$. Let $\mathcal{B}$ be the collection of bin sizes, i.e., $\mathcal{B}=\left\{b_{1}, b_{2}, \cdots, b_{m}\right\}$. For any instance $L$, let $A_{L}(m, \mathcal{B})$ and $O P T_{L}(m, \mathcal{B})$ be the total size of the bins used by an on-line algorithm $A$ and the total size of the bins used by an optimal off-line algorithm, respectively. Then the competitive ratio of algorithm $A$ is

$$
R_{A}(m, \mathcal{B})=\sup _{L} A_{L}(m, \mathcal{B}) / O P T_{L}(m, \mathcal{B})
$$

If the bin sizes are identical, i.e., $b_{1}=b_{2}=\cdots=b_{m}$, the competitive ratio is denoted as $R_{A}(m)$. Further we define the overall competitive ratios as follows.

$$
R_{A}(m, \cdot)=\sup _{\mathcal{B}} R_{A}(m, \mathcal{B}), \quad R_{A}=\sup _{m, \mathcal{B}} R_{A}(m, \mathcal{B})
$$

Known results. The extensible bin packing problem with unequal bin sizes was first studied by Dell'Olmo and Speranza [5]. They considered both the off-line case and the on-line case under the assumption that the maximum item size is not larger than the minimum bin size. They showed an upper bound of $4-2 \sqrt{2}$ for an $L P T$ (Longest Processing Time) algorithm in the off-line case and an upper bound of $5 / 4$ for a list scheduling algorithm $L S$ in the on-line case. The bound $5 / 4$ is tight in terms of the overall competitive ratio, i.e., $R_{L S}=5 / 4$.

The special case that all bin sizes are equal to one has been extensively studied. It was proved that the off-line version of the problem is $\mathcal{N} \mathcal{P}$-hard in the strong sense [4]. Actually, it can be easily reduced from the 3-partition problem [7]. Dell'Olmo et al. [4] proved that the worst-case ratio of the $L P T$ algorithm is $13 / 12$. If the number of the bins is fixed, it follows from the results of [11] that there exists a fully polynomial time approximation scheme. If $m$ is not fixed, a polynomial time approximation scheme was provided in [1]. Recently, Coffman and Lueker [3] presented a fully polynomial time asymptotic approximation scheme. They also proved that the problem does not admit a fully polynomial time approximation scheme unless $\mathcal{P}=\mathcal{N} \mathcal{P}$, when $m$ is not fixed. The on-line version was studied by Speranza and Tuza [10]. They showed that the overall competitive ratio of a list scheduling heuristic is $5 / 4$. Then a class of heuristics $H_{x}$ were presented which depend on a parameter $x, 0<x<1$, and tend to load partially loaded bins until a limit total load of $1+x$ is reached on the bin. For any number of bins, the algorithm has an overall competitive ratio bounded above by 1.228. For the lower bound of the on-line problem, they showed that no heuristic can have a competitive ratio smaller than $7 / 6$ by presenting an instance for the case that $m=2$. Ye and Zhang [12] considered on-line scheduling on a small number $m$ of bins. They proved lower bounds for $m=3,4$ and gave the competitive ratios $R_{H_{x}}(m)$ for $m=2,3$ and 4 . For $m=2$ the algorithm is best possible. An improved algorithm for $m=3$ was also presented.

A similar problem, called on-line bin stretching, was introduced by Azar and Regev [2]. A set of bins of fixed sizes is given. Items arrive on-line while it is known that there exists a valid packing of all items into the given bins. One is asked to pack all items into the bins, which can be overloaded. The goal function is the stretch factor, which is the maximum ratio for any bin between the size to which any bin was stretched (the sum of all items assigned to it) and its original size. Azar and Regev [2] studied the problem of identical bins, i.e. all original bins are of size 1. Epstein [6] considered the two-bin case with unequal bin sizes. 
Our results. In this paper, firstly we assume, as in [5], that the largest item size is at most the smallest bin size, i.e.,

$$
\max _{i=1}^{n} p_{i} \leq \min _{j=1}^{m} b_{j}
$$

We analyze the $L S$ algorithm more carefully and prove the competitive ratios for each $m$ and each collection $\mathcal{B}$ of bin sizes. The ratios differ for an even number of bins and an odd number of bins. It also shows that the competitive ratio of $L S$ for the equal bins is exactly $5 / 4$ when $m$ is even and $5 / 4-1 /\left(4 m^{2}\right)$ when $m$ is odd. We then present an improved on-line algorithm for $m=2$ and $m=3$. Finally we discuss the problem without the assumption (1). A lower bound $6 / 5$ for the overall competitive ratio is presented. We prove the competitive ratio of $L S$ for the two-bin case and present an improved on-line algorithm.

Notations and organization of the paper. Consider any algorithm $A$. During the execution of algorithm $A$, if a bin $B_{j}$ has a load (the total size of items assigned to it) at least its original size $b_{j}, B_{j}$ is called heavy; otherwise, it is called light. The difference between the load and the size $b_{j}$ is free space if $B_{j}$ is light or excess if $B_{j}$ is heavy.

Consider any instance $L$ where $\sum_{i=1}^{n} p_{i}<\sum_{j=1}^{m} b_{j}$. In an optimal packing there must be some bins with free space. Construct a new instance $L^{\prime}$ by adding new items such that no bins have free space in the optimal packing without changing the optimal value. Note that $A_{L^{\prime}}(m, \mathcal{B}) \geq A_{L}(m, \mathcal{B})$ and $O P T_{L^{\prime}}(m, \mathcal{B})=O P T_{L}(m, \mathcal{B})$. Therefore,

$$
A_{L}(m, \mathcal{B}) / O P T_{L}(m, \mathcal{B}) \leq A_{L^{\prime}}(m, \mathcal{B}) / O P T_{L^{\prime}}(m, \mathcal{B})
$$

In instance $L^{\prime}$, the total size of items is at least the total original size of bins. It implies that we only need to consider the instances in which the total size of items is at least the total original size of bins to prove an upper bound. Throughout the paper in proving the competitive ratios we always assume

$$
\sum_{i=1}^{n} p_{i} \geq \sum_{j=1}^{m} b_{j} .
$$

The remainder of the paper is organized as follows. Section 2 gives the tight bound for a list scheduling algorithm. In Section 3, we present an on-line algorithm for $m=2$ and $m=3$. We discuss the problem without the item size assumption (1) in Section 4.

\section{Tight bound for a list scheduling algorithm}

List Scheduling was introduced by Graham [8] for parallel machine scheduling. The algorithm always schedules the current job to the machine with minimum load at this time. Applying list scheduling to extensible bin packing with unequal bin sizes may result in different versions. We first consider a list scheduling algorithm, denoted by $L S$, which always assigns the incoming item to the bin of largest free space. Dell'Olmo and Speranza [5] proved that $R_{L S}=5 / 4$. In this section we figure out $R_{L S}(m, \mathcal{B})$. Then we will show that a different version of list scheduling, which always assigns the incoming item to the bin with the least load (the total size of items in a bin), is not as good as $L S$ in terms of the overall competitive ratio. The two versions of list scheduling are indeed equivalent if all the bin sizes are the same. 
Let $b_{\min }$ be the smallest bin sizes in the collection $\mathcal{B}$ and let $p_{\max }$ be the largest item size. Under the assumption (1), we have $p_{\max } \leq b_{\min }$. Consider the packing given by algorithm $L S$. For each bin $B_{j}$, let $M_{j}$ be the total size of the items it accommodates after the schedule is over. If $B_{j}$ is light, let $s_{j}$ be its free space, i.e., $s_{j}=b_{j}-M_{j}$. If $B_{j}$ is heavy, let $r_{j}$ be its free space immediately before it becomes heavy, and $e_{j}$ be its excess, i.e., $e_{j}=M_{j}-b_{j}$. We re-index the bins so that the first $k$ bins are heavy, where $k$ denotes the number of heavy bins.

Lemma 1 The competitive ratio of the list scheduling algorithm

$$
R_{L S}(m, \mathcal{B}) \geq \begin{cases}1+\frac{m b_{\min }}{4 \sum_{j=1}^{m} b_{j}}, & \text { if } m \text { is even } \\ 1+\frac{\left(m^{2}-1\right) b_{m i n}}{4 m \sum_{j=1}^{m} b_{j}}, & \text { if } m \text { is odd }\end{cases}
$$

Proof: Consider the following instances. If $m$ is even, a large number of small enough items are coming first. The total size of these items is $\sum_{j=1}^{m} b_{j}-m b_{m i n} / 2$ such that after assigning these items by $L S$, each bin has free space exactly $b_{\text {min }} / 2$. Then $m / 2$ items with size of $b_{\min }$ are coming. Clearly, the optimal value is $\sum_{j=1}^{m} b_{j}$, while $L S$ generates a total size $\sum_{j=1}^{m} b_{j}+m b_{\min } / 4$. Thus $R_{L S}(m, \mathcal{B}) \geq 1+\frac{m b_{\min }}{4 \sum_{j=1}^{m} b_{j}}$.

If $m$ is odd, the instance is slightly different. A large number of small enough items are coming first. The total size of them is $\sum_{j=1}^{m} b_{j}-(m-1) b_{m i n} / 2$, so that after assigning these items each bin has free space exactly $\frac{m-1}{2 m} b_{\text {min }}$. Then $(m-1) / 2$ items with size $b_{\min }$ are coming. Clearly, the optimal total bin size is $\sum_{j=1}^{m^{2}} b_{j}$, while $L S$ generates a total bin size of $\sum_{j=1}^{m} b_{j}+\left(m^{2}-1\right) b_{\min } /(4 m)$. Thus $R_{L S}(m, \mathcal{B}) \geq 1+\frac{\left(m^{2}-1\right) b_{m i n}}{4 m \sum_{j=1}^{m} b_{j}}$. The lemma is proved.

For an on-line algorithm in the resulting packing, if all bins are heavy or all bins are light, the packing is optimal. We only need to consider the packing with both heavy bin and light bin. It is crucial to estimate the total excess or the total free space of all bins. In proving an upper bound for algorithm $L S$ we can further assume, without loss of generality, that a bin accepts no more items once it becomes heavy. Otherwise, all bins are heavy and the packing is optimal.

Theorem 2 The competitive ratio of the list scheduling algorithm

$$
R_{L S}(m, \mathcal{B})= \begin{cases}1+\frac{m b_{\min }}{4 \sum_{j=1}^{m} b_{j}}, & \text { if } m \text { is even } \\ 1+\frac{\left(m^{2}-1\right) b_{m i n}}{4 m \sum_{j=1}^{m} b_{j}}, & \text { if } m \text { is odd }\end{cases}
$$

Proof: We only need to prove that

$$
R_{L S}(m, \mathcal{B}) \leq \begin{cases}1+\frac{m b_{\min }}{4 \sum_{j=1}^{m} b_{j}}, & \text { if } m \text { is even } \\ 1+\frac{\left(m^{2}-1\right) b_{\min }}{4 m \sum_{j=1}^{m} b_{j}}, & \text { if } m \text { is odd }\end{cases}
$$

By the algorithm $L S$, we know that

$$
\frac{\sum_{j=1}^{k} r_{j}}{k} \geq \min _{j=1, \cdots, k} r_{j} \geq \max _{j=k+1, \cdots, m} s_{j} \geq \frac{\sum_{j=k+1}^{m} s_{j}}{m-k}
$$


Then

$$
(m-k) \sum_{j=1}^{k} r_{j} \geq k \sum_{j=k+1}^{m} s_{j}
$$

From (2), we have

$$
\sum_{i=j}^{k} e_{j} \geq \sum_{j=k+1}^{m} s_{j}
$$

We add $(m-k) \sum_{j=1}^{k} e_{j}$ to both sides of the inequality 3 and from 4 , we get

$$
\begin{aligned}
(m-k) \sum_{j=1}^{k}\left(r_{j}+e_{j}\right) & \geq k \sum_{j=k+1}^{m} s_{j}+(m-k) \sum_{j=1}^{k} e_{j} \\
& \geq k \sum_{j=k+1}^{m} s_{j}+(m-k) \sum_{j=k+1}^{m} s_{j} \\
& =m \sum_{j=k+1}^{m} s_{j} .
\end{aligned}
$$

It means that $\sum_{j=k+1}^{m} s_{j} \leq \frac{(m-k)}{m} \sum_{j=1}^{k}\left(r_{j}+e_{j}\right)$. Note that the competitive ratio

$$
R_{L S}(m, \mathcal{B}) \leq\left(\sum_{i=1}^{n} p_{i}+\sum_{j=k+1}^{m} s_{j}\right) / \sum_{i=1}^{n} p_{i}
$$

Then

$$
R_{L S}(m, \mathcal{B}) \leq 1+\frac{(m-k) \sum_{j=1}^{k}\left(r_{j}+e_{j}\right)}{m \sum_{i=1}^{n} p_{i}} \leq 1+\frac{(m-k) \sum_{j=1}^{k}\left(r_{j}+e_{j}\right)}{m \sum_{j=1}^{m} b_{j}}
$$

Since $r_{j}+e_{j} \leq p_{\max } \leq b_{\min }$, we obtain

$$
R_{L S}(m, \mathcal{B}) \leq 1+\frac{(m-k) k b_{\min }}{m \sum_{j=1}^{m} b_{j}}
$$

If $m$ is even, then $(m-k) k / m^{2} \leq 1 / 4$. It follows

$$
R_{L S}(m, \mathcal{B}) \leq 1+\frac{m b_{\min }}{4 \sum_{j=1}^{m} b_{j}} .
$$

If $m$ is odd, then $(m-k) k \leq \frac{1}{4}\left(m^{2}-1\right)$. We have

$$
R_{L S}(m, \mathcal{B}) \leq 1+\frac{\left(m^{2}-1\right) b_{m i n}}{4 m \sum_{j=1}^{m} b_{j}}
$$




\section{Corollary 3}

$$
R_{L S}(m, \mathcal{B}) \leq R_{L S}(m)= \begin{cases}\frac{5}{4}, & \text { if } m \text { is even } \\ \frac{5}{4}-\frac{1}{4 m^{2}}, & \text { if } m \text { is odd }\end{cases}
$$

Moreover, $R_{L S}=5 / 4$.

Note that $R_{L S}(m, \mathcal{B})<R_{L S}(m)$ if $m \geq 2$ and $b_{i} \neq b_{j}$ for some $i, j$. It means that the competitive ratio of LS becomes smaller if the bin sizes are unequal.

Before closing this section, we consider a different version of list scheduling: assign items to the bin of least load. Denote the algorithm as $L S^{\prime}$. Consider the following simple instance $L$ for two bins. Let $b_{1}=2 b_{2}$ and let $\varepsilon>0$ be a sufficiently small number. The first two items have size of $b_{2}-\varepsilon$, which are followed by an item with size of $\varepsilon / 2$. By algorithm $L S^{\prime}$, after assigning the first two items, each bin has a load of $b_{2}-\varepsilon$. If the 3 rd item goes to the first bin $B_{1}$, an item (the last one) with size of $b_{2}$ comes, which is assigned to $B_{2}$. If the 3 rd item goes to the second bin $B_{2}$, then the 4th item has a size of $\varepsilon$ and the 5 th item (the last one) has size of $b_{2}$. The 4 th is assigned to $B_{1}$ while the 5 th is assigned to $B_{2}$. In both cases, $L S_{L}^{\prime}(2, \mathcal{B}) \geq b_{1}+b_{2}+b_{2}-\varepsilon$. In an optimal packing, we can assign the last item to $B_{2}$ and the others to $B_{1}$. Then $O P T_{L}(2, \mathcal{B})=b_{1}+b_{2}$, which shows that $R_{L S^{\prime}}(2, \mathcal{B}) \rightarrow 4 / 3$ as $\varepsilon$ tends to zero. It implies that $R_{L S^{\prime}} \geq 4 / 3$.

\section{The two- and three-bin cases}

Assume that $b_{1} \geq b_{2} \geq \cdots \geq b_{m} \geq p_{\max }$. In this section we consider the cases that $m=2$ and $m=3$. Algorithm $A_{m}(\alpha)$ : If all bins are heavy, then assign the incoming item $a_{i}$ arbitrarily. If there is a light bin whose excess will be at most $\alpha$ in case of accepting, assign the item $a_{i}$ to such a bin with lowest index; if such a bin does not exist, assign $a_{i}$ to the bin with largest free space.

In this algorithm $\alpha$ is a user-specified parameter. Choosing different parameters results in different algorithms.

Lemma 4 For any parameter $\alpha>0$, the competitive ratio of algorithm $A_{2}(\alpha)$ is at least $1+b_{2} /\left(3\left(b_{1}+\right.\right.$ $\left.\left.b_{2}\right)\right)$.

Proof: Let $N$ be a sufficiently large integer. If $\alpha<b_{2} / 3$, consider an instance $L$ as follows. The first $\left\lfloor\left(b_{1}-2 b_{2} / 3\right) N\right\rfloor$ items are small items, each with size $1 / N$, which are followed by items $a_{1}, a_{2}, a_{3}$ with size of $b_{2} / 3,2 b_{2} / 3$ and $2 b_{2} / 3$, respectively. Clearly, $A_{2}(\alpha)$ assigns all the small items together with $a_{1}$ to bin $B_{1}$, and $a_{2}$ to bin $B_{2}$. Thus no matter where $a_{3}$ is assigned, $A_{2}(\alpha)_{L}(2, \mathcal{B}) \geq b_{1}+b_{2}+b_{2} / 3-1 / N$. For an optimal solution, we can assign $a_{1}$ and $a_{2}$ to $B_{2}$, and the remaining items to $B_{1}$. Thus $O P T_{L}(2, \mathcal{B})=$ $b_{1}+b_{2}$, which follows that $A_{2}(\alpha)_{L}(2, \mathcal{B}) / O P T_{L}(2, \mathcal{B}) \rightarrow 1+b_{2} /\left(3\left(b_{1}+b_{2}\right)\right)$ as $N$ goes to infinity.

If $\alpha \geq b_{2} / 3$, consider the following instance $L$ : the first $\left\lfloor\left(b_{1}-2 b_{2} / 3\right) N\right\rfloor$ items with size $1 / N$, are followed by item $a_{1}$ with size $b_{2}$. So $A_{2}(\alpha)_{L}(2, \mathcal{B}) \geq b_{1}+b_{2} / 3+b_{2}-1 / N$. In an optimal packing, we assign $a_{1}$ to $B_{2}$, and the remaining items to $B_{1}$. $O P T_{L}(2, \mathcal{B})=b_{1}+b_{2}$, which follows $A_{2}(\alpha)_{L}(2, \mathcal{B}) / O P T_{L}(2, \mathcal{B}) \rightarrow 1+b_{2} /\left(3\left(b_{1}+b_{2}\right)\right)$ as $N$ tends to infinity.

By setting $\alpha=b_{2} / 3$, we prove that the competitive ratio of $A_{2}(\alpha)$ is $1+b_{2} /\left(3\left(b_{1}+b_{2}\right)\right)$. 
Theorem 5 The competitive ratio of $A_{2}(\alpha)$ is $1+b_{2} /\left(3\left(b_{1}+b_{2}\right)\right)$ if $\alpha=b_{2} / 3$. Moreover the overall competitive ratio is $7 / 6$.

Proof: If both bins are light or both are heavy, the packing is optimal. We only need to consider the case that exactly one bin is heavy. From the assumption (2), we have $M_{1}+M_{2} \geq b_{1}+b_{2}$, where $M_{i}$ is the load of bin $B_{i}, i=1,2$.

Let $\alpha=b_{2} / 3$. Let $T$ be the excess of the heavy bin and let $X$ be the free space of the light bin. If $T \leq \alpha$, then $R_{A_{2}(\alpha)}(2, \mathcal{B}) \leq\left(T+b_{1}+b_{2}\right) /\left(b_{1}+b_{2}\right) \leq 1+b_{2} /\left(3\left(b_{1}+b_{2}\right)\right)$. Now assume that $T>\alpha$. We want to prove $X \leq \alpha$.

Case 1. $B_{2}$ is light. $X=b_{2}-M_{2}$. Let $a_{n}$ be the last item assigned to $B_{1}$, which makes the excess over $\alpha$. $B_{2}$ is not empty and before $a_{n}$ is assigned $B_{1}$ is light and has an free space at least $X$. Otherwise, $a_{n}$ should have been assigned to $B_{2}$. It implies that the size of the items in $B_{2}$ is larger than $X+\alpha$, otherwise all the items in $B_{2}$ can be assigned to $B_{1}$. Thus $X=b_{2}-M_{2}<b_{2}-(X+\alpha)$. It follows that $X<\alpha$.

Case 2. $B_{1}$ is light. $X=b_{1}-M_{1}$. Before the last item is assigned to $B_{2}, B_{2}$ is light and has an free space at least $X$. The total size of items in $B_{2}$ before the last item is assigned is at most $b_{2}-X$. According to the algorithm, $b_{2}-X+M_{1}>b_{1}+\alpha$. Then $b_{2}-X>b_{1}-M_{1}+\alpha$ which implies that $X \leq \alpha$.

In both cases we have $R_{A_{2}(\alpha)}(2, \mathcal{B}) \leq\left(X+M_{1}+M_{2}\right) /\left(M_{1}+M_{2}\right) \leq 1+b_{2} /\left(3\left(b_{1}+b_{2}\right)\right)$. It is easy to verify that $R_{A_{2}(\alpha)}(2, \cdot)=7 / 6$.

Since no on-line algorithm can have an overall competitive ratio less than 7/6 for two bins [5], $A_{2}(\alpha)$ is an optimal on-line algorithm for $m=2$ in terms of the overall competitive ratio, setting $\alpha=b_{2} / 3$.

We will show a lower bound for two bins under the assumption (1). Let $b_{1}=k b_{2} / 3+x$, where $0 \leq x<b_{2} / 3$ and $k \geq 3$ ( $k$ is an integer).

Lemma 6 No on-line algorithm can achieve a competitive ratio smaller than

$$
R= \begin{cases}1+\left(b_{2} / 3-x\right) /\left(b_{1}+b_{2}\right), & \text { if } 0 \leq x \leq b_{2} / 6 \\ 1+x /\left(b_{1}+b_{2}\right), & \text { if } b_{2} / 6<x<b_{2} / 3\end{cases}
$$

Proof: Let $A$ be any on-line algorithm. Consider the following instance $L$. The items with size of $b_{2} / 3$ come one by one until $n_{2}=2$ or $n_{1}=k-1$, where $n_{i}$ is the number of items with size of $b_{2} / 3$ placed into bin $B_{i}$ by algorithm $A$ for $i=1,2$.

Case 1. $n_{2}=0, n_{1}=k-1$. If $0 \leq x \leq b_{2} / 6$, the next two items have size of $2 b_{2} / 3$. Thus $A_{L}(2, \mathcal{B}) \geq$ $b_{1}+b_{2}+b_{2} / 3-x$.

If $b_{2} / 6<x<b_{2} / 3$, the next item has size of $x$. If $x$ goes to $B_{1}$, two items with size of $2 b_{2} / 3$ come. It follows that $A_{L}(2, \mathcal{B}) \geq b_{1}+b_{2}+b_{2} / 3$. If $x$ goes to $B_{2}$, an item with size of $b_{2}$ comes. Then $A_{L}(2, \mathcal{B}) \geq b_{1}+b_{2}+x$.

Case 2. $n_{2}=1, n_{1}=k-1$. The next item has size of $b_{2}$ and then $A_{L}(2, \mathcal{B}) \geq b_{1}+b_{2}+b_{2} / 3$.

Case 3. $n_{2}=2, n_{1}=p$, where $0 \leq p<k-1$. 
Subcase 3a For the sake of proof, we also consider the case $p=k-1$. In this case the next item has size of $2 b_{2} / 3+x$, and thus $A_{L}(2, \mathcal{B}) \geq b_{1}+b_{2}+b_{2} / 3$.

Subcase 3b If $p=k-2$, we consider two subcases. If $0 \leq x \leq b_{2} / 6$, the next two items have sizes of $x$ and $b_{2}$ follows. If the first item is assigned to $B_{1}$, then $A_{L}(2, \mathcal{B}) \geq b_{1}+b_{2}+b_{2} / 3$. If the first item is assigned to $B_{2}$, then we have $A_{L}(2, \mathcal{B}) \geq b_{1}+b_{2}+b_{2} / 3-x$. If $b_{2} / 6<x<b_{2} / 3$, the next two items have size of $b_{2} / 3+x$ and $2 b_{2} / 3$. So, $A_{L}(2, \mathcal{B}) \geq b_{1}+b_{2}+x$.

Subcase 3c If $p=k-3$, the next two items have size of $2 b_{2} / 3+x / 2 . A_{L}(2, \mathcal{B}) \geq b_{1}+b_{2}+b_{2} / 3$.

Subcase 3d If $p \leq k-4$, let $s=\lceil(k-p) / 3\rceil-1$. The next $s$ items have size of $b_{2}$. If one of such items is packed into $B_{2}$, then $A_{L}(2, \mathcal{B}) \geq b_{1}+b_{2}+2 b_{2} / 3$. Otherwise, all the $s$ items go to $B_{1}$. The free space of $B_{1}$ becomes $(k-p) b_{2} / 3+x-(\lceil(k-p) / 3\rceil-1) b_{2}$, which is either $b_{2} / 3+x$ or $2 b_{2} / 3+x$ or $b_{2}+x$. Then it is reduced to the above three subcases $3 \mathrm{a}-3 \mathrm{c}$.

Note that in any of the above cases, $O P T_{L}(2, \mathcal{B})=b_{1}+b_{2}$. Thus, the lemma is proved.

Lemma 6 shows that $R_{A}(2, \mathcal{B}) \geq 1+\frac{b_{2}}{6\left(b_{1}+b_{2}\right)}$ for any on-line algorithm $A$. It also shows that algorithm $A_{2}(\alpha)$ is optimal for the case that $x=0$ (or $b_{1}=k b_{2} / 3$ for some integer $k \geq 4$ ), by setting $\alpha=b_{2} / 3$. Now we consider the three-bin case.

Lemma 7 For any parameter $\alpha>0$, the competitive ratio of algorithm $A_{3}(\alpha)$ is at least $1+b_{3} /\left(2\left(b_{1}+\right.\right.$ $\left.\left.b_{2}+b_{3}\right)\right)$.

Proof: Let $N$ be a sufficiently large integer. If $\alpha \geq b_{3} / 2$, consider the following instance $L$. The first $\left\lfloor b_{1} N-1\right\rfloor$ items with size of $1 / N$ are followed by an item of size $b_{3} / 2+1 / N$. Clearly, $A_{3}(\alpha)_{L}(3, \mathcal{B}) \geq$ $b_{1}+b_{2}+3 b_{3} / 2-1 / N$ and $O P T_{L}(3, \mathcal{B}) \leq b_{1}+b_{2}+b_{3}+1 / N$. It follows that $A_{3}(\alpha)_{L}(3, \mathcal{B}) / O P T_{L}(3, \mathcal{B}) \rightarrow$ $1+b_{3} /\left(2\left(b_{1}+b_{2}+b_{3}\right)\right)$ as $N$ tends to infinity.

If $\alpha<b_{3} / 2$, consider the following instance $L .\left\lfloor b_{1} N-1\right\rfloor$ items with size of $1 / N$ are followed by item $a_{1}$ with size of $\alpha+1 / N$. Then $\left\lfloor\left(b_{2}-b_{3} / 2\right) N\right\rfloor+1$ items with size of $1 / N$ are coming, which are followed by the last two items $a_{2}, a_{3}$ with size of $b_{3} / 2, b_{3}-\alpha-1 / N$, respectively. Algorithm $A_{3}(\alpha)$ assigns the first $\left\lfloor b_{1} N-1\right\rfloor$ items together with $a_{1}$ to $B_{1},\left\lfloor\left(b_{2}-b_{3} / 2\right) N+1\right\rfloor$ items with size $1 / N$ to $B_{2}$, and $a_{2}$ and $a_{3}$ to $B_{3}$. It shows $A_{3}(\alpha)_{L}(3, \mathcal{B}) \geq b_{1}+b_{2}+3 b_{3} / 2-2 / N$. In an optimal packing, we can assign $a_{1}$ and $a_{3}$ to $B_{3}$, the first $\left\lfloor b_{1} N-1\right\rfloor$ items with size $1 / N$ to $B_{1}$ and the remaining items to $B_{2}$. It follows that $O P T_{L}(3, \mathcal{B}) \leq b_{1}+b_{2}+b_{3}$. Then $A_{3}(\alpha)_{L}(3, \mathcal{B}) / O P T_{L}(3, \mathcal{B}) \rightarrow 1+b_{3} /\left(2\left(b_{1}+b_{2}+b_{3}\right)\right)$, as $N$ tends to infinity.

In the following, we prove that the competitive ratio of algorithm $A_{3}(\alpha)$ can reach the lower bound $1+b_{3} /\left(2\left(b_{1}+b_{2}+b_{3}\right)\right)$ by setting $\alpha=b_{3} / 2$.

Theorem 8 The competitive ratio of the algorithm $A_{3}(\alpha)$ is $1+b_{3} /\left(2\left(b_{1}+b_{2}+b_{3}\right)\right)$ if $\alpha=b_{3} / 2$.

Proof: Let $\alpha=b_{3} / 2$. In the packing given by $A_{3}(\alpha)$, let $M_{i}$ be the total size of the items assigned to bin $B_{i}$. If all the bins are heavy or all the bins are light, the algorithm gives an optimal packing. Hence, we only need to consider the following cases. 
Case 1. Only one bin is heavy. If the excess of the heavy bin is at most $\alpha$, we can get the competitive ratio $1+b_{3} /\left(2\left(b_{1}+b_{2}+b_{3}\right)\right)$ immediately. Before the last item of the heavy bin is assigned, all the three bins are light and the heavy bin has the largest free space at the moment.

Subcase 1a $B_{1}$ is heavy. Then $M_{1}>b_{1}+\alpha$. Note that $M_{2}+M_{3}>b_{2}+\alpha$. We have

$$
\begin{aligned}
R_{A_{3}(\alpha)}(3, \mathcal{B}) & \leq\left(M_{1}+b_{2}+b_{3}\right) /\left(M_{1}+M_{2}+M_{3}\right) \\
& =1+\left(b_{2}+b_{3}-M_{2}-M_{3}\right) /\left(M_{1}+M_{2}+M_{3}\right) \\
& \leq 1+\left(b_{2}+b_{3}-b_{2}-\alpha\right) /\left(b_{1}+b_{2}+b_{3}\right) \\
& \leq 1+b_{3} /\left(2\left(b_{1}+b_{2}+b_{3}\right)\right)
\end{aligned}
$$

Subcase 1b $B_{2}$ is heavy. $M_{2}>b_{2}+b_{3} / 2$ and $M_{1}+M_{3}>b_{1}+b_{3} / 2$.

$$
\begin{aligned}
R_{A_{3}(\alpha)}(3, \mathcal{B}) & \leq\left(M_{2}+b_{1}+b_{3}\right) /\left(M_{1}+M_{2}+M_{3}\right) \\
& =1+\left(b_{1}+b_{3}-M_{1}-M_{3}\right) /\left(M_{1}+M_{2}+M_{3}\right) \\
& \leq 1+b_{3} /\left(2\left(M_{1}+M_{2}+M_{3}\right)\right) \\
& \leq 1+b_{3} /\left(2\left(b_{1}+b_{2}+b_{3}\right)\right)
\end{aligned}
$$

Subcase 1c $B_{3}$ is heavy. $M_{3}>b_{3}+b_{3} / 2$. Let $X$ be the total free space of $M_{1}$ and $M_{2}$. Then $X=$ $b_{1}+b_{2}-\left(M_{1}+M_{2}\right)$. Let $Y_{3}$ be the load in $B_{3}$ before the last item is assigned. Thus

$$
b_{3}-Y_{3} \geq b_{2}-M_{2}, b_{3}-Y_{3} \geq b_{1}-M_{1} .
$$

We have $X=b_{1}+b_{2}-\left(M_{1}+M_{2}\right) \leq 2\left(b_{3}-Y_{3}\right)$. On the other hand,

$$
Y_{3}+M_{1} \geq b_{1}+b_{3} / 2, Y_{3}+M_{2} \geq b_{2}+b_{3} / 2 .
$$

We have $X=b_{1}+b_{2}-\left(M_{1}+M_{2}\right) \leq 2 Y_{3}-b_{3}$. So, $X \leq \min \left\{2\left(b_{3}-Y_{3}\right), 2 Y_{3}-b_{3}\right\} \leq b_{3} / 2$. Therefore,

$$
\begin{aligned}
R_{A_{3}(\alpha)}(3, \mathcal{B}) & \leq\left(X+M_{1}+M_{2}+M_{3}\right) /\left(M_{1}+M_{2}+M_{3}\right) \\
& \leq 1+b_{3} /\left(2\left(b_{1}+b_{2}+b_{3}\right)\right) .
\end{aligned}
$$

Case 2. Only one bin is light. If the free space $X$ of the light bin is at most $\alpha$ or the total excess of the two heavy bins is at most $\alpha$, we have the competitive ratio immediately. We only need to consider the case that $X>\alpha$ and the total excess exceeds $\alpha$. However, we will show that it is impossible. Before considering the following two cases, we assume that $M_{i}>0$ for $i=1,2,3$. Otherwise, the total excess is at most $\alpha$.

Subcase 2a $B_{3}$ is light. $M_{3}=b_{3}-X<\alpha=b_{3} / 2$. Let $Y_{i}$ be the load of $B_{i}$ immediately before it becomes heavy, for $i=1,2$. Then $b_{i}-Y_{i} \geq X$; otherwise the last item, which makes the total excess of $B_{1}$ and $B_{2}$ over $\alpha$, would have been assigned to bin $B_{3}$, then any item less than $b_{3}$ can be assigned to $B_{1}$ or $B_{2}$. Thus all the items in $B_{3}$ should have been assigned to $B_{i}$ by the algorithm. It is a contradiction. 
Subcase 2b $B_{3}$ is heavy. Recall that any item size is at most $b_{3}$. There are at least two items in $B_{3}$. Assume that $a_{k}$ is the last item assigned to $B_{3}$. Those items assigned to $B_{3}$ have size at least $X>b_{3} / 2$. Thus the idle space of $B_{3}$ before $a_{k}$ is assigned is less than $X$, which means that $a_{k}$ would have not been assigned to $B_{3}$. It is a contradiction.

From Theorems 5 and 8 we realize that the competitive ratio of algorithm $A_{m}(\alpha)$ for unequal bins is better than that for equal bins.

\section{Without the assumption on item sizes}

In this section, we will discuss the problem without the assumption (1) for the two-bin case. However, it is reasonable to assume that all items with size at most $b_{1}$.

Lemma 9 Without the assumption (17), no deterministic on-line algorithms can achieve an overall competitive ratio lower than $6 / 5$ for two bins.

Proof: Let $b_{1}=3 / 2$ and $b_{2}=1$. The first item $a_{1}$ has size of $1 / 2$. If $a_{1}$ is assigned to bin $B_{2}$, two items $a_{2}, a_{3}$ with size of 1 are coming. If $a_{1}$ is assigned to bin $B_{1}$, item $a_{2}$ with size of $3 / 2$ is coming. For both cases, the overall competitive ratio is at least $6 / 5$.

Lemma 10 For any on-line algorithm A the competitive ratio

$$
R_{A}(2, \mathcal{B}) \geq \begin{cases}1+\left(4 b_{2} / 3-b_{1}\right) /\left(b_{1}+b_{2}\right), & \text { if } b_{1} \leq 7 b_{2} / 6, \\ 1+\left(b_{1}-b_{2}\right) /\left(b_{1}+b_{2}\right), & \text { if } 7 b_{2} / 6<b_{1}<4 b_{2} / 3 \\ 1+b_{2} /\left(3\left(b_{1}+b_{2}\right)\right), & \text { if } b_{1} \geq 4 b_{2} / 3\end{cases}
$$

Proof: Let $A$ be any on-line algorithm. Consider first the case that $b_{1} \geq 4 b_{2} / 3$ with the following instance $L$. The first two items have size of $b_{2} / 3$. If both items go to $B_{1}$, the next item has size of $b_{1}$; if both items go to $B_{2}$, two items with size of $2 b_{2} / 3$ and $b_{1}-b_{2} / 3$, respectively, are coming; if the first two items go to different bins, the next item has size of $b_{1}$. For any of the above cases, $A_{L}(2, \mathcal{B}) \geq b_{1}+4 b_{2} / 3$ and the optimal value $O P T_{L}(2, \mathcal{B})=b_{1}+b_{2}$. It implies that the lemma is true.

Now turn to the case that $b_{1}<4 b_{2} / 3$. Let $b_{1}=b_{2}+x$, where $0 \leq x<b_{2} / 3$. The lemma follows from Lemma 6 by setting $k=3$.

Now we turn to upper bounds. Assume that all items have size of at most $b_{1}\left(b_{1} \geq b_{2}\right)$.

Theorem 11 The competitive ratio of the list scheduling algorithm $L S$ for two bins is $1+\min \left\{b_{2}, b_{1} / 2\right\} /\left(b_{1}+\right.$ $\left.b_{2}\right)$.

Proof: We first show the upper bound. Without loss of generality, we consider the case only one bin is heavy.

Case 1. $b_{1} / 2 \geq b_{2}$. The $L S$ algorithm assigns items to the bin with largest free space. Recall that the total size of items is not less than the total size of bins, we get that the free space is at most $b_{2}$. Then, $L S_{L}(2, \mathcal{B}) \leq \sum p_{i}+b_{2}, O P T_{L}(2, \mathcal{B}) \geq \sum p_{i} \geq b_{1}+b_{2}$, which implies $R_{L S}(2, \mathcal{B}) \leq$ $1+b_{2} /\left(b_{1}+b_{2}\right)$. 
Case 2. $b_{1} / 2<b_{2}$. Let $X$ be the free space and $T$ be the excess. We only consider the case that both $X$ and $T$ are greater than $b_{1} / 2$. Otherwise, it follows that $R_{L S}(2, \mathcal{B}) \leq 1+b_{1} /\left(2\left(b_{1}+b_{2}\right)\right)$. Let $p_{n}$ be the size of the last item assigned to the bin which has an excess over $b_{1} / 2$. Recall that all items have size of at most $b_{1}$. Let $Y_{i}$ be the load of bin $B_{i}$ before the last item is assigned. Then $b_{i}-Y_{i} \geq X>b_{1} / 2$. Since $p_{n} \leq b_{1}$, if $X>b_{1} / 2$, then $T$ is less than $b_{1} / 2$, which implies that either $X$ or $T$ is at most $b_{1} / 2$.

The following simple instance shows that the bound is tight. Consider three items with sizes of $\min \left\{b_{2}, b_{1} / 2\right\}, \max \left\{0, b_{2}-b_{1} / 2\right\}$, and $b_{1}$, respectively. The optimal value is $b_{1}+b_{2}$ while $L S$ costs $b_{1}+b_{2}+\min \left\{b_{2}, b_{1} / 2\right\}$.

In the following, we present an on-line algorithm to improve the upper bound in some cases.

\section{Algorithm $A 2$ :}

- If $b_{1} \leq 4 b_{2} / 3$, apply algorithm $A_{2}(\alpha)$ by setting $\alpha=b_{2} / 3$;

- if $4 b_{2} / 3<b_{1} \leq 2 b_{2}$, apply $A_{2}(\alpha)$ by setting $\alpha=b_{1}-b_{2}$;

- if $b_{1}>2 b_{2}$, apply algorithm $L S$.

Theorem 12 The competitive ratio of algorithm $A 2$ is

$$
R_{A 2}(2, \mathcal{B}) \leq \begin{cases}1+b_{2} /\left(3\left(b_{1}+b_{2}\right)\right), & \text { if } b_{1} \leq 4 b_{2} / 3 \\ 1+\left(b_{1}-b_{2}\right) /\left(b_{1}+b_{2}\right), & \text { if } 4 b_{2} / 3<b_{1} \leq 2 b_{2} \\ 1+b_{2} /\left(b_{1}+b_{2}\right), & \text { if } b_{1}>2 b_{2}\end{cases}
$$

\section{Proof:}

Consider the following cases:

Case 1. $b_{1} \leq 4 b_{2} / 3$. We use $A_{2}(\alpha)$, by setting $\alpha=b_{2} / 3$. The proof is similar as the one of Theorem 5 .

Case 2. $4 b_{2} / 3<b_{1} \leq 2 b_{2}$. We only need to consider the case that exactly one bin is heavy, the excess of the heavy bin is over $b_{1}-b_{2}$, and the free space of the light bin is larger than $b_{1}-b_{2}$. However, we will show this case is impossible. Before the last item $a_{n}$, which makes the excess over $b_{1}-b_{2}$, is assigned, if $B_{2}$ is empty, then the excess is at most $b_{1}-b_{2}$ after assigning $a_{n}$. Assume that $B_{2}$ is not empty before $a_{n}$ is assigned. Note that $B_{1}$ has an free space larger than $b_{1}-b_{2}$ before $a_{n}$ is assigned. It means that the total size of items in $B_{2}$ is more than $2\left(b_{1}-b_{2}\right)$. Then the idle space of bin $B_{2}$ is at most $b_{2}-2\left(b_{1}-b_{2}\right)<\left(b_{1}-b_{2}\right)$. In this case $a_{n}$ is assigned to $B_{1} . B_{2}$ is a light bin and the idle space is less than $b_{1}-b_{2}$. It is a contradiction.

Case 3. $b_{1}>2 b_{2}$. It follows from Theorem 11 
Final Remarks. There are many open questions. Although we have proved that the algorithm $A_{2}(\alpha)$ is optimal in terms of the overall competitive ratio, it is still open to find a best possible on-line algorithm for two bins in terms of the competitive ratio. It is interesting to design an algorithm with a better competitive ratio than $L S$ algorithm for any number of bins. The problem without the assumption (1) becomes more difficult. We only proved the competitive ratio of $L S$ for the two-bin case. Any improvement on the lower bounds is also of interest.

\section{Acknowledgements}

The authors would like to thank the referees and the Associate Editor for their helpful suggestions.

\section{References}

[1] N. Alon, Y. Azar, G.J. Woeginger, and T. Yadid. Approximation schemes for scheduling on parallel machines. Journal of Scheduling 1:55-66, 1998.

[2] Y. Azar and O. Regev. On-line bin-stretching. Theoretical Computer Science 268:17-41, 2001.

[3] E.G. Coffman and G.S. Lueker. Approximation algorithms for extensible bin packing. Proceedings of the twelfth Annual ACM-SIAM Symposium on Discrete Algorithms, 586-588, 2001.

[4] P. Dell'Olmo, H. Kellerer, and M.G. Speranza, Z. Tuza. A 13/12 approximation algorithm for bin packing with extendable bins. Information Processing Letters 65:229-233, 1998.

[5] P. Dell'Olmo and M.G. Speranza. Approximation algorithms for partitioning small items in unequal bins to minimize the total size. Discrete Applied Mathematics 94:181-191, 1999.

[6] L. Epstein. Bin stretching revisited. Acta Informatica 39:97-117, 2003.

[7] M.R. Garey and D.S. Johnson. Computers and Intractability: A guide to the Theory of $\mathcal{N} \mathcal{P}$ completeness. W.H.Freeman, San Francisco, 1979.

[8] R. Graham. Bounds for certain multiprocessing anomalies. Bell System Technical Journal 45:1563$1581,1966$.

[9] J. Sgall. On-line scheduling - A survey, Online Algorithms: The State of the Art, eds. A. Fiat and G. J. Woeginger. Lecture Notes in Comput. Sci. 1442, 196-231, Springer-Verlag, 1998.

[10] M.G. Speranza and Z. Tuza. On-line approximation algorithms for scheduling tasks on identical machines with extendable working time. Annals of Operations Research 86:494-506, 1999.

[11] G.J. Woeginger. When does a dynamic programming formulation guarantee the existence of an FPTAS? In Proceedings of the tenth Annual ACM-SIAM Symposium on Discrete Algorithms, 820-829, 1999.

[12] D. Ye and G. Zhang. On-line scheduling with extendable working time on a small number of machines. Information Processing Letters 85:171-177, 2003. 